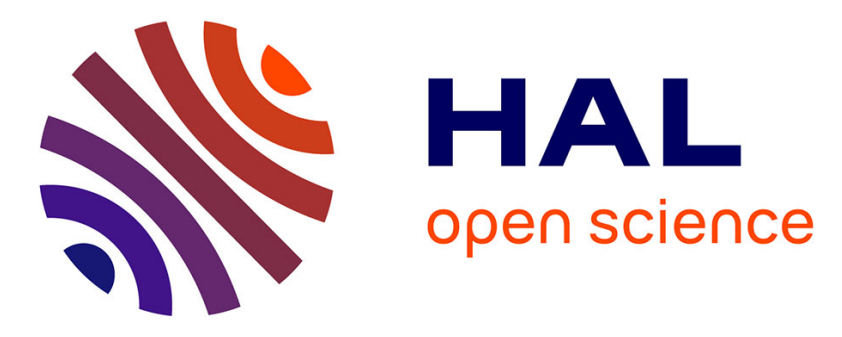

\title{
Highlighting the Container Memory Consolidation Problems in Linux
}

Francis Laniel, Damien Carver, Julien Sopena, Franck Wajsburt, Jonathan

Lejeune, Marc Shapiro

\section{- To cite this version:}

Francis Laniel, Damien Carver, Julien Sopena, Franck Wajsburt, Jonathan Lejeune, et al.. Highlighting the Container Memory Consolidation Problems in Linux. NCA 2019 - 18th IEEE International Symposium on Network Computing and Applications, Sep 2019, Cambridge, United States. pp.1-4, 10.1109/NCA.2019.8935034 . hal-02424007

\section{HAL Id: hal-02424007 https://hal.science/hal-02424007}

Submitted on 26 Dec 2019

HAL is a multi-disciplinary open access archive for the deposit and dissemination of scientific research documents, whether they are published or not. The documents may come from teaching and research institutions in France or abroad, or from public or private research centers.
L'archive ouverte pluridisciplinaire HAL, est destinée au dépôt et à la diffusion de documents scientifiques de niveau recherche, publiés ou non, émanant des établissements d'enseignement et de recherche français ou étrangers, des laboratoires publics ou privés. 


\title{
Highlighting container memory consolidation problem in Linux
}

\author{
Francis Laniel $^{\ddagger}$, Damien Carver ${ }^{\ddagger}$, Julien Sopena ${ }^{\ddagger}$, Franck Wajsburt*, Jonathan Lejeune ${ }^{\ddagger}$ and Marc Shapiro ${ }^{\ddagger}$ \\ ${ }^{\ddagger}$ Sorbonne Université, CNRS, Inria, LIP6, DELYS Team, F-75005 Paris, France \\ *Sorbonne Université, CNRS, LIP6, F-75005 Paris, France \\ *łfirstname.lastname@lip6.fr
}

\begin{abstract}
The container mechanism supports server consolidation; to ensure memory performance isolation, Linux relies on static memory limits. However, this results in poor performance, because an application needs are dynamic. In this article we will show current problems with memory consolidation for containers in Linux.

Index Terms-Linux, container, memory, memory consolidation
\end{abstract}

\section{INTRODUCTION}

This paper is about showing the problem of memory consolidation for containers in Linux.

Logical servers are consolidated by running several of them on a single physical machine, thereby saving money. The logical servers should remain mutually isolated, i.e. execution of one logical server should not impact any other.

One popular consolidation mechanism, in Linux is called "containers" [1]-[4]. A container is a group of processes isolated from the others. Containers are advantageous for security (e.g. a file that belongs to a container can not be read from another), deployment (e.g. running a container is as simple as running a shell command) and resource control (e.g. a container can be restricted to, say, a specific CPU core) [5].

This article focuses specifically on container memory consolidation, which consists of multiplexing the physical memory of the machine across the virtual memory demands of all containers. Memory consolidation can be effective if, at every point in time, the total memory demands of all containers is less than the available physical memory, which is the common case, because data center utilization usually remains well under $100 \%$ [6], [7].

The classical virtual memory mechanism ensures security isolation. However, it is insufficient for memory performance isolation (ensuring to each container enough memory for it to perform well) because it does not stop one container from starving the others of physical memory. Furthermore, the metrics available to the kernel (e.g. frequency of page faults, I/O requests, use of CPU cycle, etc.) are not suitable for performance isolation, because they are not directly relevant to performance as experienced from the application perspective, which is better characterized by, for instance latency or throughput.

To avoid a container starving the others, the system administrator can limit the total amount of its physical memory. If it would exceed its limit, some of its memory will be reclaimed, making it available to others. In practice, the kernel tends to reclaim preferably from the file page cache; this causes a performance decrease for containers that access files [8]. The limits to container size are static, and do not adapt to the containers' dynamic behaviors. This is problematic, because it is challenging to estimate the optimal amount of memory for an application to execute smoothly [9], [10].

Our main contribution consists in an experimental demonstration of the limitations of the existing Linux mechanisms.

We organize the remainder of our paper as follows. Section II presents some technical background. Section III shows the issues with existing Linux mechanisms. Finally, we conclude and discuss future work in Section IV.

\section{TECHNICAL BACKGROUND}

A container is represented using the Linux cgroup mechanism [11]-[14]. A cgroup is a set of processes whose collective usage of resources is limited. In particular, the total amount of physical memory used across all the processes of a memory cgroup is capped by the max and soft limits described hereafter. In the rest of this section, we study how Linux manages the memory of containers under memory pressure, i.e. when free physical memory is scarce.

\section{A. No limits}

When no limits are set and under memory pressure, memory will be reclaimed from all containers. If containers have different memory needs, the kernel will allocate memory to satisfy them, even as their needs change during execution. Indeed, if a container uses, at some moment of its execution, less memory than earlier the kernel will reallocate the unused memory to another container. This constitutes memory consolidation; however there will not be memory performance isolation. Indeed, if a container uses less its memory it will lose it, because it will be reclaimed. In summary, when no limits are set, memory consolidation occurs at the expense of memory performance isolation.

\section{B. The max and soft limit mechanism}

Linux has two mechanisms to control memory reclamation of containers. The first is the max limit; a container will not be allocated a memory footprint larger than its max limit, even if there exists unused memory elsewhere. If a process needs physical memory, and its container has already reached its max 
limit, the kernel may reallocate memory from another process of the same container, but not from another container. This mechanism avoids situations where some containers would starve the others.

The soft limit is similar to the max limit, except that it is active only when there is memory pressure and it is only besteffort. The intent is that the system administrator will set a soft limit approximating the container's Working Set (WS) size [15]. Unfortunately, it is hard to estimate WS size [9], [10].

In other words, the size of a container is limited to its soft limit when there is memory pressure, otherwise, to its max limit. However, these limits are not directly related to the containers' current memory needs; they may be higher (impeding memory consolidation) or lower (impeding memory performance isolation).

\section{MEMORY CONSOLIDATION VS. MEMORY PERFORMANCE ISOLATION}

\section{A. Reference experiment}

In order to demonstrate the issues experimentally, we first run a reference experiment. This experiment consists of executing a benchmark, detailed hereafter, to obtain reference number for throughput and memory footprint. The throughput was obtained from the benchmark output while memory and inputs were collected by docker. We measure the max throughput, under high offered load, to be 800 transactions per second. The reference memory footprint was measured to be $2.8 \mathrm{~GB}$. These numbers are pictured in Figure 1 to Figure 3 as horizontal green lines. In the scenario that we will describe in next section, the high offered load corresponds to the generation of 800 transactions per second. We defined low offered load as what is required to reach 200 transactions per second. This number is showed as horizontal black line in Figure 1a to Figure $3 a$.

\section{B. Experimental scenario}

We run an experiment whose goal is to show that without limits there is no memory performance isolation, and that the max and soft limits impede memory consolidation. Our experimental scenario has two containers, A and B, which run an OLTP workload and experience changes in activity. We expect that, when both containers have high offered load, memory performance isolation should occur. When one container has high offered load and the other has low offered load or is stopped, memory consolidation should allow it to reach its reference performance. Specifically, the experiment has 6 steps:

$\varphi_{1} \mathrm{~A}$ and $\mathrm{B}$ both have high offered loads. Containers will bring database records in memory but physical memory available is inferior to databases' sizes. So, there will be memory pressure. Containers will then have to access the disk so their throughputs will be low.

$\varphi_{2}$ The high offered load continues for A, and it decreases for B. If memory consolidation is effective A should be able to take memory from $\mathrm{B}$, and increase its throughput to the reference value.

$\varphi_{3}$ B has high offered load. This step is similar to step 1. So, containers will have a low throughput.

$\varphi_{4}$ Both containers have low offered loads. We expect that they will be able to answer to all the transactions.

$\varphi_{5}$ B has high offered load, not A. This step is symmetric to step 2.

$\varphi_{6} \mathrm{~A}$ is completely stopped. B still has high offered load. We expect $B$ to perform the same as the reference.

Each step of the scenario lasts for 180 seconds.

\section{Experimental environment}

Our experimental machine is a workstation with an Intel®Xeon®E5-1603 clocked at $2.8 \mathrm{GHz}, 8 \mathrm{~GB}$ of DDR3 and a SSD. To create memory pressure, we run our experiments in a virtual machine (VM) restricted to $4 \mathrm{CPU}$ cores and $3 \mathrm{~GB}$ of memory.

We use qemu 2.8.1 as VM hypervisor, docker 18.09.6 and its python library docker-py 3.7 .2 to manage containers. Our containers execute the benchmark sysbench oltp. We modified this benchmark to dynamically change the rate of transactions generated. The benchmark makes request to a database managed by mysql 5.7 . We run our experiments on Linux 4.19.

Our two containers (A and B) run with 2 cores each. They each read a database of $4 \mathrm{~GB}$. Since our VM has only $3 \mathrm{~GB}$ of memory, the two databases will not fit in memory and some accesses to those databases will not be cached by Linux page cache resulting in physical I/O, which heavily decrease the throughput.

Then, we run the scenario described in Section III-B 10 times and compute the mean and standard deviation every second. Each point in curves depicted in Figure 1, Figure 2 and Figure 3 is the mean and its associated standard deviation for this second across the 10 runs.

\section{Experiment with no limits set}

We run the experiment described in Section III-B without setting any limits. By doing so, we wish to demonstrate that all containers are reclaimed equally, and there is no memory performance isolation. Our results are depicted in Figure 1.

Figure 1a shows the containers' throughputs over time and Figure 1b depicts their memory footprints. In Figure 1a, the throughput of a container that has high offered load increases at the expense of one with low offered load $\left(\varphi_{2}\right.$ and $\left.\varphi_{5}\right)$. Figure $1 \mathrm{~b}$ shows that the memory footprint of the container with high offered load increases, while the container with low offered load shrinks. Memory consolidation is taking place but imperfectly, since an active container never reaches the reference level of performance. When A stops, B's footprint grows, so its performance increases, reaching the reference level $\left(\varphi_{6}\right)$. When both containers have high offered loads they have the same throughput because they have the same memory footprint, due to the lack of memory performance isolation $\left(\varphi_{1}\right.$ and $\left.\varphi_{3}\right)$. 


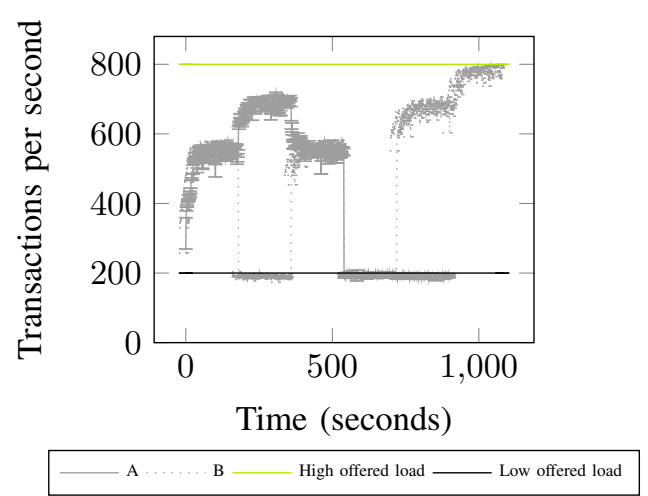

(a) Throughput

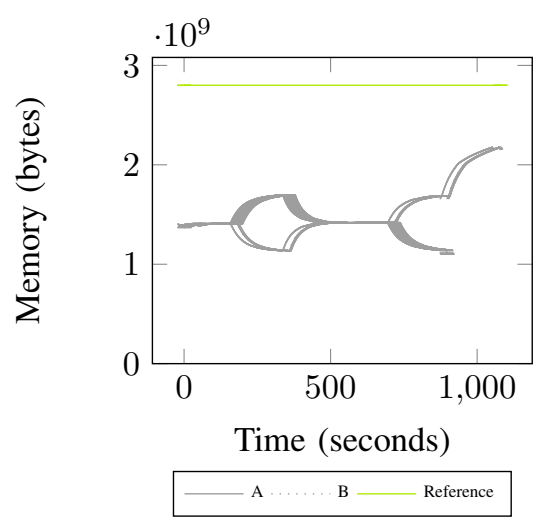

(b) Physical memory footprint

Fig. 1: No limits set

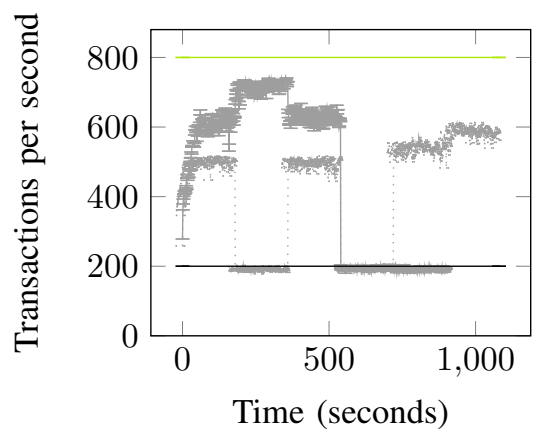

(a) Throughput

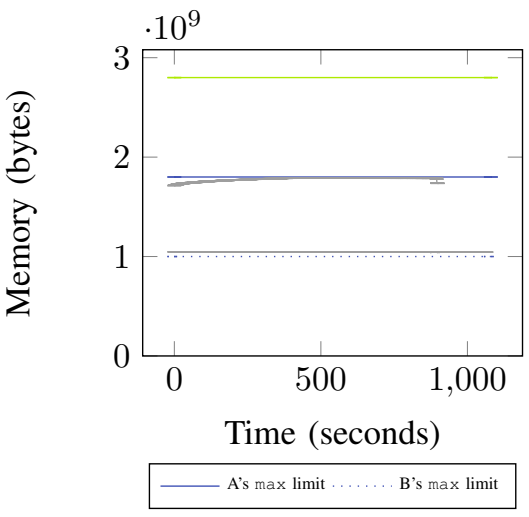

(b) Physical memory footprint

Fig. 2: Max limits set to $1.8 \mathrm{~GB}(\mathrm{~A})$ and $1 \mathrm{~GB}(\mathrm{~B})$

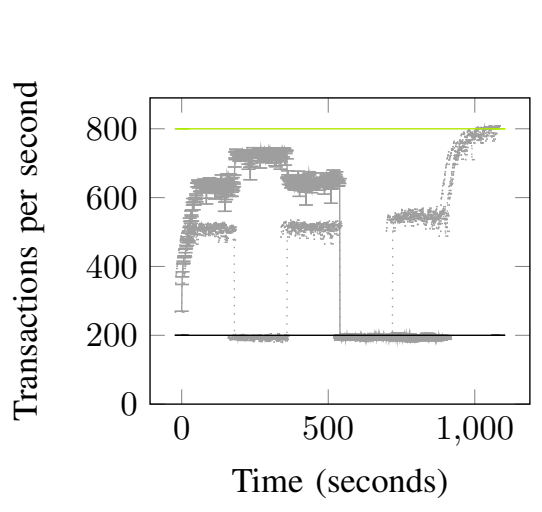

(a) Throughput

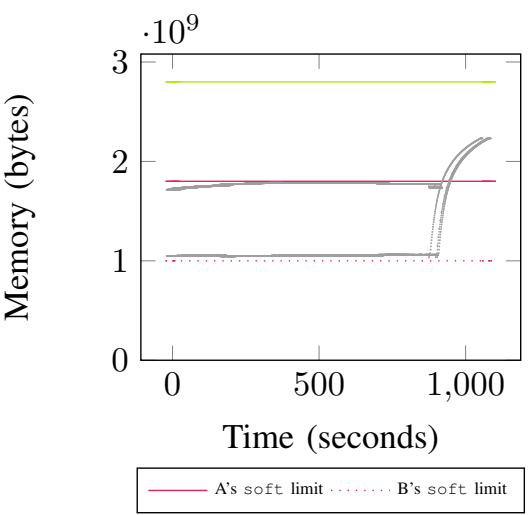

(b) Physical memory footprint

Fig. 3: Soft limits set to $1.8 \mathrm{~GB}$ (A) and $1 \mathrm{~GB}$ (B) 


\section{E. Experiment with max limit}

We demonstrate that max limit impedes memory consolidation, by running the same experiment with max limits set to $1.8 \mathrm{~GB}$ for $\mathrm{A}$ and $1 \mathrm{~GB}$ for $\mathrm{B}$. Those values were chosen such that their total equals the reference memory footprint.

In Figure 2a, A has better performance than B. This can be explained by looking at Figure $2 \mathrm{~b}$. Because the memory footprints reach the max limits, A has more memory than $\mathrm{B}$; therefore it will be able to answer more requests $\left(\varphi_{1}\right.$ and $\left.\varphi_{3}\right)$. Figure $2 \mathrm{~b}$ shows that there is no memory consolidation, because even when B has low offered load, A's memory footprint does not increase, and it never reaches the reference level $\left(\varphi_{2}\right)$. Conversely, when A has low offered load and B has high offered load, B's memory does not grow, because of its max limit $\left(\varphi_{5}\right)$. Therefore, B reaches only 500 to 550 transactions per second. Worst, when A stops, B is not able to expand its memory footprint and experiences little increase in performance $\left(\varphi_{6}\right)$.

To conclude, with the max limit there is memory performance isolation, since A acquires more memory than $\mathrm{B}$, but memory consolidation is impeded.

\section{F. Experiment with soft limit}

Under memory pressure, the soft limit has a similar effect to the max limit, also impeding memory consolidation. We show this behavior by executing the experiment using soft limits. Figure 3 shows that the results are nearly the same as above. This is expected because during our experiment there is memory pressure, and containers will tend to remain under the soft limits, which equal the previous max limits. Note however how, in $\varphi_{6}$, B's footprint increases; because A was stopped, relaxing memory pressure, the soft limit mechanism is not activated anymore, allowing B to increase its memory allocation.

To summarize, the soft limit mechanism supports memory performance isolation (B has less memory than A) under memory pressure. This limits memory consolidation but when memory pressure disappears, memory consolidation becomes effective again.

\section{G. Summary}

Our study shows that the existing mechanisms are not effective at memory consolidation and memory performance isolation. Without limits, there is weak memory consolidation but no memory performance isolation. The max limit supports memory performance isolation, but impedes memory consolidation. The soft limit is analogous to the max limit under memory pressure, but when memory is plentiful it does enable memory consolidation. We summarize these results in Table I.

\section{CONCLUSION AND FUTURE WORKS}

In this paper, we showed the issues of memory consolidation $v s$. memory performance isolation for existing mechanisms with containers. Our experiments showed without limits, there is a weak memory consolidation, but no memory performance

\begin{tabular}{|l|c|c|}
\hline Mechanism used & Memory consolidation & Memory performance isolation \\
\hline no limits & weak & no \\
\hline max limit & no & yes \\
\hline soft limit & no & yes \\
\hline
\end{tabular}

TABLE I: Summary of memory consolidation and memory performance isolation of existing mechanisms

isolation since containers have the same memory footprints. The max limit offers memory performance isolation but consolidation is impeded. The soft limit, which activates only under memory pressure, acts similarly to the max limit.

In future work, we will extend the soft limit mechanism to consolidate memory. Our mechanism will be based on applicative probe to give information about containers performance to the kernel.

\section{REFERENCES}

[1] Amazon, "Amazon Elastic Container Service." [Online]. Available: https://aws.amazon.com/ecs/?nc1=h_1s

[2] Microsoft, "Microsoft Web App for Containers." [Online]. Available: https://azure.microsoft.com/en-us/services/app-service/containers/

[3] Alibaba, "Alibaba Container Service." [Online]. Available: https://www.alibabacloud.com/product/container-service?spm= a2c5t.10695662.1996646101.searchclickresult.55a3212bnXyv1v

[4] OVH, "OVH Kubernetes." [Online]. Available: https://www.ovh.com/fr/ kubernetes/

[5] Docker Inc., "What is a Container?" [Online]. Available: https: //www.docker.com/resources/what-container

[6] D. Meisner, B. T. Gold, and T. F. Wenisch, "PowerNap: eliminating server idle power," ACM SIGARCH Computer Architecture News, vol. 37, no. 1, p. 205, Mar. 2009. [Online]. Available: http: //dl.acm.org/citation.cfm?doid=2528521.1508269

[7] L. A. Barroso, J. Clidaras, and U. Hölzle, "The Datacenter as a Computer: An Introduction to the Design of WarehouseScale Machines, Second edition," Synthesis Lectures on Computer Architecture, vol. 8, no. 3, pp. 1-154, Jul. 2013. [Online]. Available: http://www.morganclaypool.com/doi/abs/10.2200/ S00516ED2V01Y201306CAC024

[8] The kernel development community, "Concepts overview." [Online]. Available: https://www.kernel.org/doc/html/latest/admin-guide/ $\mathrm{mm} /$ concepts.html

[9] B. Gregg, "Working Set Size Estimation," Feb. 2018. [Online] Available: http://www.brendangregg.com/wss.html

[10] V. Nitu, A. Kocharyan, H. Yaya, A. Tchana, D. Hagimont, and H. Astsatryan, "Working Set Size Estimation Techniques in Virtualized Environments: One Size Does not Fit All," Proceedings of the ACM on Measurement and Analysis of Computing Systems, vol. 2, no. 1, pp. 1-22, Apr. 2018. [Online]. Available: http: //dl.acm.org/citation.cfm?doid=3203302.3179422

[11] K. Hiroyu, "Cgroup And Memory Resource Controller," Nov. 2008. [Online]. Available: https://www.static.linuxfound.org/jp_uploads/ seminar20081119/CgroupMemcgMaster.pdf

[12] Rami Rosen, "Namespace and cgroups, the basis of Linux containers," Seville, Spain, Feb. 2016. [Online]. Available: https://www.netdevconf. org/1.1/proceedings/slides/rosen-namespaces-cgroups-lxc.pdf

[13] Linux, "Memory Resource Controller." [Online]. Available: https: //www.kernel.org/doc/Documentation/cgroup-v1/memory.txt

[14] Zhenyun Zhuang, Cuong Tran, J. Weng, H. Ramachandra, and B. Sridharan, "Taming memory related performance pitfalls in linux Cgroups," in 2017 International Conference on Computing, Networking and Communications (ICNC). Silicon Valley, CA, USA: IEEE, Jan. 2017, pp. 531-535. [Online]. Available: http: //ieeexplore.ieee.org/document/7876184/

[15] P. J. Denning, "The working set model for program behavior," in Proceedings of the ACM symposium on Operating System Principles SOSP '67. Not Known: ACM Press, 1967, pp. 15.1-15.12. [Online]. Available: http://portal.acm.org/citation.cfm?doid $=800001.811670$ 